\title{
Sustainable Production of Dihydroxybenzene Glucosides Using Immobilized Amylosucrase from Deinococcus geothermalis ${ }^{\text {s }}$
}

\author{
Hun Sang Lee ${ }^{1 \dagger}$, Tae-Su Kim ${ }^{1 \dagger}$, Prakash Parajuli ${ }^{1}$, Ramesh Prasad Pandey ${ }^{1,2}$, and Jae Kyung Sohng ${ }^{1,2 *}$ \\ ${ }^{1}$ Institute of Biomolecule Reconstruction (iBR), Department of Life Science and Biochemical Engineering, Sun Moon University, Asan 31460, \\ Republic of Korea \\ ${ }^{2}$ Department of Pharmaceutical Engineering and Biotechnology, Sun Moon University, Asan 31460, Republic of Korea
}

\author{
Received: May 23, 2018 \\ Revised: June 15, 2018 \\ Accepted: July 4, 2018 \\ First published online \\ July 3, 2018 \\ *Corresponding author \\ Phone: +82-41-530-2246; \\ Fax: +82-41-530-8229; \\ E-mail: sohng@sunmoon.ac.kr \\ ${ }^{\dagger}$ These authors contributed \\ equally to this work.
}

S upplementary data for this paper are available on-line only at http://jmb.or.kr.

pISSN 1017-7825, eISSN 1738-8872

Copyright(C) 2018 by

The Korean Society for Microbiology and Biotechnology

\begin{abstract}
The amylosucrase encoding gene from Deinococcus geothermalis DSM 11300 (DgAS) was codonoptimized and expressed in Escherichia coli. The enzyme was employed for biosynthesis of three different dihydroxybenzene glucosides using sucrose as the source of glucose moiety. The reaction parameters, including temperature, $\mathrm{pH}$, and donor (sucrose) and acceptor substrate concentrations, were optimized to increase the production yield. This study demonstrates the highest ever reported molar yield of hydroquinone glucosides $325.6 \mathrm{mM}$ $(88.6 \mathrm{~g} / \mathrm{l})$, resorcinol glucosides $130.2 \mathrm{mM}$ (35.4 g/l) and catechol glucosides $284.4 \mathrm{mM}$ (77.4 g/l) when $400 \mathrm{mM}$ hydroquinone, $200 \mathrm{mM}$ resorcinol and $300 \mathrm{mM}$ catechol, respectively, were used as an acceptor substrate. Furthermore, the use of commercially available amyloglucosidase at the end of the transglycosylation reaction minimized the glucooligosaccharides, thereby enhancing the target productivity of mono-glucosides. Moreover, the immobilized DgAS on Amicogen LKZ118 beads led to a $278.4 \mathrm{mM}(75.8 \mathrm{~g} / 1), 108.8 \mathrm{mM}$ $(29.6 \mathrm{~g} / \mathrm{l})$ and $211.2 \mathrm{mM}(57.5 \mathrm{~g} / \mathrm{l})$ final concentration of mono-glycosylated product of hydroquinone, catechol and resorcinol at 35 cycles, respectively, when the same substrate concentration was used as mentioned above. The percent yield of the total glycosides of hydroquinone and catechol varied from $85 \%$ to $90 \%$ during 35 cycles of reactions in an immobilized system, however, in case of resorcinol the yield was in between $65 \%$ to $70 \%$. The immobilized DgAS enhanced the efficiency of the glycosylation reaction and is therefore considered effective for industrial application.
\end{abstract}

Keywords: Dihydroxybenzene glucosides, amylosucrase, amyloglucosidase, Deinococcus geothermalis, enzyme immobilization

\section{Introduction}

Amylosucrase (AS) is a glycosyltransferase (GT) structurally belonging to the glycoside hydrolase $[1,2]$. Transglycosylation reactions of AS are usually conjugations of single and/or multiple (gluco-oligosaccharides) sugar(s) with a great advantage compared with Leloir GTs. For production of bioactive phenolic glycosides [2,3], AS has been used in the synthesis of a variety of attractive biomaterials including amylose-like polymers, starch, dendritic nanoparticles, and microparticle encapsulation [4-6]. Although AS has potential application in glycodiversification, it is rarely reported for transglycosyation of phenolic compounds.

The biologically active phenolic compounds usually contain single or multiple functional groups such as hydroxyl and methyl moieties bonded to aromatic hydrocarbons. These hydroxyl groups are prone to subsequent modifications, which alters their physiochemical functions compared with the parent compounds. Glycosides of hydroquinone (paradihydroxybenzene), popularly known as skin-whitening agents and widely used in the cosmetic industry, inhibit melanin formation in the skin and lighten dark skin, freckles and age spots, as well as repair sun damage [7]. Resorcinol and catechol are derivatives of meta- and ortho- 
dihydroxybenzenes, respectively. They also exhibit potent biological activities and therefore have pharmaceutical as well as cosmeceutical applications. Resorcinol is used as a skin-whitening agent and as an ointment to relieve pain and itching [8-10], while catechol is used as an anti-cancer compound and as a cosmeceutical agent $[4,11]$. Although these phenolic compounds are highly useful, their applications have been limited due to issues related to stability, poor solubility, and short life in a photosensitive environment. Bulky sugar attachment to these compounds has been shown to resolve these issues of water solubility, stability, and biological properties [12].

In this study, three dihydroxybenzenes (hydroquinone, catechol and resorcinol) were glycosylated using the amylosucrase enzyme prepared using a codon optimized gene of Deinococcus geothermalis DSM 11300 (DgAS). The oligosaccharide-conjugated phenolic glycosides were also generated during transglycosylation. The productions of these phenolic mono-glucosides were enhanced by optimization of various reaction parameters, enzyme immobilization and treatment with commercial amyloglucosidase from Aspergillus niger. Previous studies reported the synthesis of $\alpha$-arbutin and its potential use in the cosmeceutical industry $[13,14]$.

\section{Materials and Methods}

\section{Chemicals and Reagents}

Hydroquinone, resorcinol, catechol, ascorbic acid, sucrose, and amyloglucosidase (from Aspergillus niger) were purchased from Sigma-Aldrich (USA). High performance liquid chromatography (HPLC) grade acetonitrile and water were purchased from Mallinckrodt Baker (USA). Restriction enzymes were purchased from Takara Bio (Japan) and Promega (USA). Amicogen LKZ118 beads were purchased from Amicogen (Korea)

\section{Bacterial Strains, Cloning and Culture Conditions}

Escherichia coli XL1 blue was used as a host for all DNA manipulation while E. coli BL21 (DE3) (Novagen, Darmstadt, Germany) was used for the expression and production of DgAS enzyme using pHCE IIB (NdeI) vector. Recombinant strains were grown in Luria-Bertani (LB) agar plate and broth medium supplemented with ampicillin $(100 \mu \mathrm{g} / \mathrm{ml})$ at $37^{\circ} \mathrm{C}$. The nucleotide sequence of amylosucrase (GenBank Accession No. ABF44874.1) sourced from Deinococcus geothermalis DSM 11300 was codonoptimized and synthesized by General Biosystems (USA). The 1,984-bp gene fragment was flanked by NdeI and HindIII restriction sites.

\section{Enzyme Expression and Purification}

Recombinant E. coli BL21 (DE3) harboring DgAS was cultured in $50 \mathrm{ml} \mathrm{LB}$ broth at $37^{\circ} \mathrm{C}$ for $24 \mathrm{~h}$. The cells were harvested by centrifugation at $840 \times g$ (for $10 \mathrm{~min}$ at $4^{\circ} \mathrm{C}$ and washed using a buffer containing $50 \mathrm{mM}$ Tris- $\mathrm{HCl}$ and $10 \%$ glycerol $(\mathrm{pH} 7.5)$. These cells were re-suspended in the same buffer and disrupted with a sonicator in an ice bath. The supernatant and cell debris were separated by centrifugation at $13,475 \times g$ for $15 \mathrm{~min}$ at $4^{\circ} \mathrm{C}$. Expressed protein was analyzed by sodium dodecyl sulfatepolyacrylamide gel electrophoresis (SDS-PAGE) with 12\% (w/v) acrylamide gel. The supernatant was purified using nickel nitrilotriacetic acid (Ni-NTA) affinity column chromatography (Qiagen Inc., USA) and eluted at various concentrations (10, 100, 200 , and $500 \mathrm{mM}$ ) of imidazole, respectively, as previously reported $[15,16]$.

Enzymatic Reaction Conditions for DgAS and Amyloglucosidase The transglycosylation reaction was performed using dihydroxybenzenes (hydroquinone, resorcinol and catechol) (Sigma-Aldrich, USA) as acceptor molecules in the presence of sucrose as glucose donor catalyzed by amylosucrase (DgAS). The $200 \mu$ of the total reaction mixture contained $20 \mathrm{mM}$ dihyroxybenzene, $2 \mathrm{mM}$ ascorbic acid, $200 \mathrm{mM}$ sucrose, $0.4 \mathrm{mg} / \mathrm{ml}$ amylosucrase enzyme and $200 \mathrm{mM}$ Tris- $\mathrm{HCl}$ buffer at $\mathrm{pH}$ 7. The reactions were incubated at $37^{\circ} \mathrm{C}$ for $30 \mathrm{~min}$ and stopped by keeping the reaction mixture in a boiling water bath for $10 \mathrm{~min}$. The reaction mixtures were treated with $13 \mathrm{U} / \mathrm{ml}$ of commercial amyloglucosidase (Sigma-Aldrich, USA) at $40^{\circ} \mathrm{C}$ for $3 \mathrm{~h}$. The samples were treated with chilled methanol to stop further reaction and centrifuged at $13,475 \times g$ for $15 \mathrm{~min}$ to separate them from protein precipitates. The reaction mixtures were analyzed by high performance liquid chromatography-photo diode array (HPLC-PDA) and confirmed by electrospray ionization mass spectrometry (ESI/MS) and nuclear magnetic resonance (NMR) analyses. The production yield was enhanced by the addition of $10 \%$ of various antioxidants (ascorbic acid) to the reaction mixtures.

\section{Analytical Methods}

Reverse-phase HPLC-PDA analyses was performed at $280 \mathrm{~nm}$ UV absorbance using a $\mathrm{C}_{18}$ column packed with YMC-Pack ODS$\mathrm{AQ} ; 4.6 \mathrm{~mm}$ internal diameter, $150 \mathrm{~mm}$ long and $5 \mu \mathrm{m}$ particle size long, connected to photo diode array (PDA) on binary condition consisting of solvent A ( $0.05 \%$ trifluoroacetic acid buffer-TFA), water, and $100 \%$ acetonitrile maintained at a flow rate of $1 \mathrm{ml} / \mathrm{min}$ for a 25-min program. The acetonitrile was set throughout as $0-$ $40 \%$ (0-15 min), $40-75 \%$ ( $15-20 \mathrm{~min}$ ), $75-0 \%$ (20-25 min). The purification of compounds was carried out by preparative HPLC with a $C_{18}$ column (YMC-PACK ODS-AQ; 150-250 mm long, $20 \mathrm{~mm}$ internal diameter, $10 \mu \mathrm{m}$ particle size) connected to a UV detector using a 36-min binary program. The flow was $10 \mathrm{ml} / \mathrm{min}$ throughout the program as acetonitrile flowed for $0-20 \mathrm{~min}$ (40\%), 20-30 $\mathrm{min}(75 \%), 30-36 \mathrm{~min}(0 \%)$.

The high resolution quadruple-time of flight electrospray ionization-mass spectrometry (HRQTOF-ESI/MS) was performed in positive- ion mode using Acquity mass spectrometer (with 
UPLC; Waters, USA) coupled with a Synapt G2-S system (Waters). The purified compounds were structurally determined by Avance II 300 Bruker (Germany) BioSpin NMR spectrometer equipped with a TCI CryoProbe $(5 \mathrm{~mm})$. All the samples were exchanged with $\mathrm{D}_{2} \mathrm{O}$ and dissolved in methanol- $d_{4}$ for ${ }^{1} \mathrm{H}$ NMR (proton), ${ }^{13} \mathrm{C}$ NMR (carbon).

\section{Bioconversion of Phenolic Compounds and Purification of Reaction Products}

An identical DgAS transglycosylation was carried out in the presence of three dihydroxybenzenes according to the optimized parameters listed in the supplementary section. To enhance the production yield and substrate stability, the selected antioxidant ( $10 \%$ ascorbic acid) was added to the reaction mixture followed by $13 \mathrm{U} / \mathrm{ml}$ of glucosidase enzyme resulting in the highest yield. The reaction was maintained at optimized donor and acceptor substrate concentrations, temperature, and $\mathrm{pH}$ for $30 \mathrm{~min}$. The large preparative scale reaction was carried out and purified using preparative HPLC in binary condition as mentioned in the analytical section.

\section{Optimization of Transglycosylation Reaction Conditions}

The transglycosylation reaction was optimized by studying the required concentration of donor and acceptor substrates in different ratio, temperature and $\mathrm{pH}$. The identical reactions were carried out in the presence of reaction components as mentioned above varying the ratio of phenolic compounds and sucrose from 2.5:1, 5:1, 10:1, 15:1, and 20:1 followed by incubation at $37^{\circ} \mathrm{C}$ for $30 \mathrm{~min}$. For temperature optimization, reaction mixtures were incubated in ranges from $35^{\circ} \mathrm{C}$ to $60^{\circ} \mathrm{C}$ while to compare reactivity according to $\mathrm{pH}$, the reaction mixtures were made in varying buffer conditions of $\mathrm{pH} 5$ to $\mathrm{pH} 9$. Each time, the reaction mixtures were analyzed by HPLC-PDA to determine the optimized parameters based percentage production yields.

\section{Immobilization of Amylosucrase}

The enzyme immobilization experiment was performed using AMICOGEN LKZ118 beads. The beads were washed three times with distilled water followed by dehydration for $40 \mathrm{sec}$ using a vacuum pump. Around $5 \mathrm{~g}$ of beads in an Erlenmeyer flask was mixed in $10.2 \mathrm{ml}$ of distilled water followed by addition of $1.1 \mathrm{M}$ $\mathrm{Na}_{2} \mathrm{SO}_{4}$. This solution was dissolved and incubated at $25^{\circ} \mathrm{C}$ at 100 $\mathrm{rpm}$. Around $70 \mathrm{mg}$ of enzyme per gram of beads were mixed and immobilized at $25^{\circ} \mathrm{C}$ at $100 \mathrm{rpm}$ for $24 \mathrm{~h}$. After immobilization, it was washed using distilled water three times and dehydrated again for $40 \mathrm{sec}$ through a vacuum pump before being stored at $4^{\circ} \mathrm{C}$.

The immobilized enzyme reactions were carried out at $40^{\circ} \mathrm{C}$ for $30 \mathrm{~min}$. The reaction mixture was vigorously shaked ( $200 \mathrm{rpm})$ throughout the reaction incubation time. The reactions were carried out in $5 \mathrm{ml}$ volume containing $200 \mathrm{mM}$ Tris- $\mathrm{HCl}$ ( $\mathrm{pH} 7.0$ ), $2 \mathrm{M}$ sucrose, $400 \mathrm{mM}$ hydroquinone, $40 \mathrm{mM}$ ascorbic acid and $300 \mathrm{mg}$ of DgAS-immobilized beads. Identical reactions were carried out with resorcinol and catechol, but the concentration of sucrose, acceptor substrate, and ascorbic acid was different. For resorcinol, $1 \mathrm{M}$ sucrose, $200 \mathrm{mM}$ resorcinol, and $20 \mathrm{mM}$ ascorbic acid were used which was $1.5 \mathrm{M}$ sucrose, $300 \mathrm{mM}$ catechol, and $20 \mathrm{mM}$ ascorbic acid in the reaction mixture of catechol. The immobilization rate was calculated from the initial and final concentrations of immobilized enzymes. These enzymes were washed and reacted repeatedly 50 times, which also suggests the efficiency of the technique and phenolic glycoside synthesis in this experiment.

\section{Results}

\section{DgAS Expression and Purification}

The codon-optimized and synthesized DgAS gene (supplementary S1) was cloned into pHCE IIB (NdeI) with $6 \times$ histidine tagged at its carboxyl terminus. The cloned gene of $1.984 \mathrm{~Kb}$ was confirmed by restriction digestion with NdeI/HindIII enzymes. The gene was expressed in E. coli BL21(DE3). The expressed soluble fraction of the protein was purified as described in methods and analyzed by SDS-PAGE analysis which showed protein of $72.49 \mathrm{kDa}$ resembling the 6x-his tagged fused DgAS (Fig. S1).

\section{Transglycosylation of Phenolic Compounds}

Amylosucrase (DgAS) enzyme was used for enzymatic catalysis in the presence of sucrose as a sugar donor. Hydroquinone, resorcinol and catechol were used as substrates. The aim of this work was to synthesize a monoglucoside derivative of each substrate using an inexpensive glucose donor source catalyzed by the glucosyltransferase activity of DgAS. Characteristically, this enzyme hydrolyzes sucrose and transfers glucose moiety to the hydroxyl group of phenolic substrates during glycosylation [13, 17].

The HPLC-PDA analysis of the reaction mixture of all three dihydroxybenzenes showed glucosylation of each substrate to generate multiple products. The standard hydroquinone that appeared at a retention time $\left(t_{\mathrm{R}}\right)$ of $8.2 \mathrm{~min}$ was converted to mono and multiple products appeared below $t_{\mathrm{R}}$ of $6.6 \mathrm{~min}$ (Figs. 1A and 1B). Similar results were observed with resorcinol $t_{\mathrm{R}}=10.4 \mathrm{~min}$ (Figs. $1 \mathrm{C}$ and 1D) and catechol $t_{\mathrm{R}} 11 \mathrm{~min}$ as a mono-glucoside (Figs. 1E and 1F). Multi-glucoside products of resorcinol and catechol appeared at $t_{\mathrm{R}}=7.2$ to $t_{\mathrm{R}}=8.4 \mathrm{~min}$ and $t_{\mathrm{R}}=8.2$ to $t_{\mathrm{R}}=10.8 \mathrm{~min}$, respectively. Minor products conjugated with gluco-oligosaccharides (glucotriose, glucotetraose, glucopentaose, etc) attached to hydroquinone, resorcinol and catechol were detected along with mono-glucosides of each substrate (Fig. 1). The conjugation of multiple sugar units into aglycons was confirmed by high resolution HRQTOF-ESI/MS (Fig. S2). 


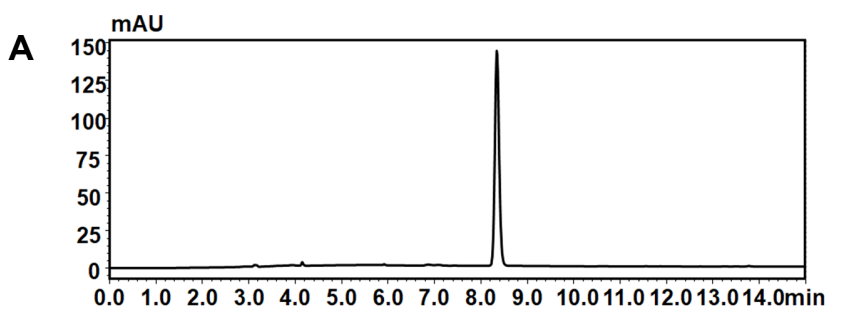

mAU
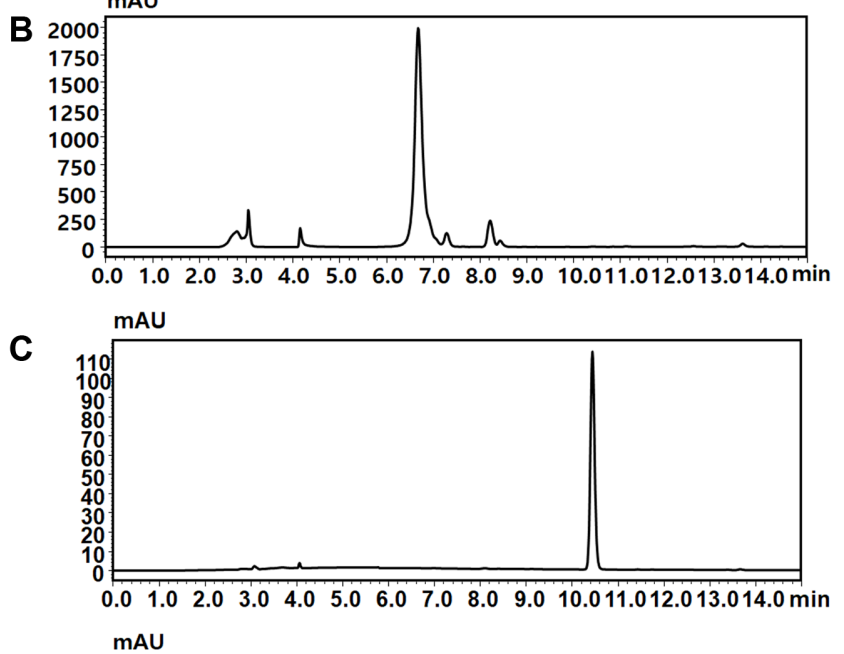

D

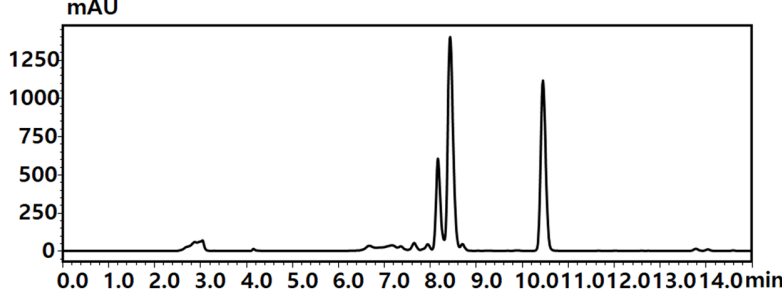

E
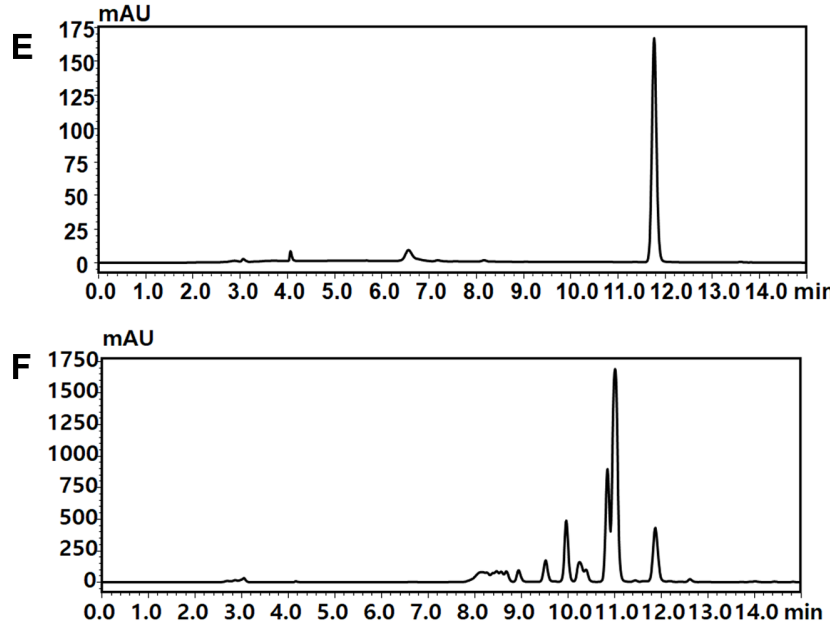

Fig. 1. HPLC-PDA chromatogram of transglycosylation reactions of hydroquinone, resorcinol and catechol compared with standards.

(A) Hydroquinone standard (B) Hydroquinone reaction (C) Resorcinol standard (D) Resorcinol reaction (E) Catechol standard (F) Catechol reaction.

\section{Optimization of Sucrose Concentration, Temperature and pH}

Different concentrations of sucrose ranging from 50 to $400 \mathrm{mM}$ were added in the reaction mixtures of hydroquinone, resorcinol and catechol, keeping $20 \mathrm{mM}$ of each substrate and other components constant. Enhanced production occurred with optimization of the maximum donor (sucrose) substrate concentration yielded the highest target products. By using $20 \mathrm{mM}$ hydroquinone as the substrate for transglycosylation, the hydroquinone monoglucoside (arbutin) increased with the rise in sucrose concentration up to $300 \mathrm{mM}$, and the production was constant upon further increase of the sucrose to $400 \mathrm{mM}$ (Fig. 2A). The conversion rate of hydroquinone was $95.1 \%$ when 300-400 mM of sucrose was used. Similarly, in the case of resorcinol, the synthesis of resorcinol mono-glucoside and oligosaccharide-conjugated resorcinol (multi-glucosides) occurred. The synthesis of mono-glucoside increased continuously up to $200 \mathrm{mM}$ of sucrose concentration and it remained stable up to $400 \mathrm{mM}$ whereas the production of oligosaccharide-conjugated resorcinol derivatives continued to increase until the donor substrate concentration increased to $400 \mathrm{mM}$ (Fig. 2B). When catechol was used as the substrate, the formation of catechol mono-glucoside (mono) increased up to $100 \mathrm{mM}$ of sucrose concentration followed by eventual decline in the conversion percentage (Fig. 2C). Concurrently, the oligosaccharide-conjugated catechol gradually increased up to a sucrose concentration of 400 $\mathrm{mM}$ (Fig. 2C). These results suggested that minimization of the synthesis of the oligosaccharide-conjugated products of resorcinol and catechol by sugar hydrolyzing enzymes increased the production of mono-glucosides.

The reactions were carried out using $200 \mathrm{mM}$ Tris- $\mathrm{HCl}$ buffer ( $\mathrm{pH} 7.0$ ), $2 \mathrm{mM}$ ascorbic acid, $100 \mathrm{mM}$ sucrose, and $20 \mathrm{mM}$ of phenolic compounds at varying incubation temperatures ranging from $35^{\circ} \mathrm{C}$ to $60^{\circ} \mathrm{C}$. The optimal temperature for transglycosylation reaction was found to be $40^{\circ} \mathrm{C}$ for hydroquinone (Fig. 2D). The highest conversion level was observed at $35{ }^{\circ} \mathrm{C}$ with resorcinol and catechol. However, for $\mathrm{pH}$ optimization, different ranges of $\mathrm{pH}$ buffer were used along with other reaction components. The reactions were incubated at $40^{\circ} \mathrm{C}$. Reactions with hydroquinone $(98 \%$ conversion) and resorcinol $(43.6 \%$ conversion) yielded the highest products at $\mathrm{pH} \sim 7$ while the yield was the highest at $\mathrm{pH} 8.0$ in case of catechol (60.6\% conversion) (Figs. 2G-2I).

\section{Improved Transglycosylation by Amyloglucosidase}

Amyloglucosidase hydrolyzes the glucosidic bonds from 

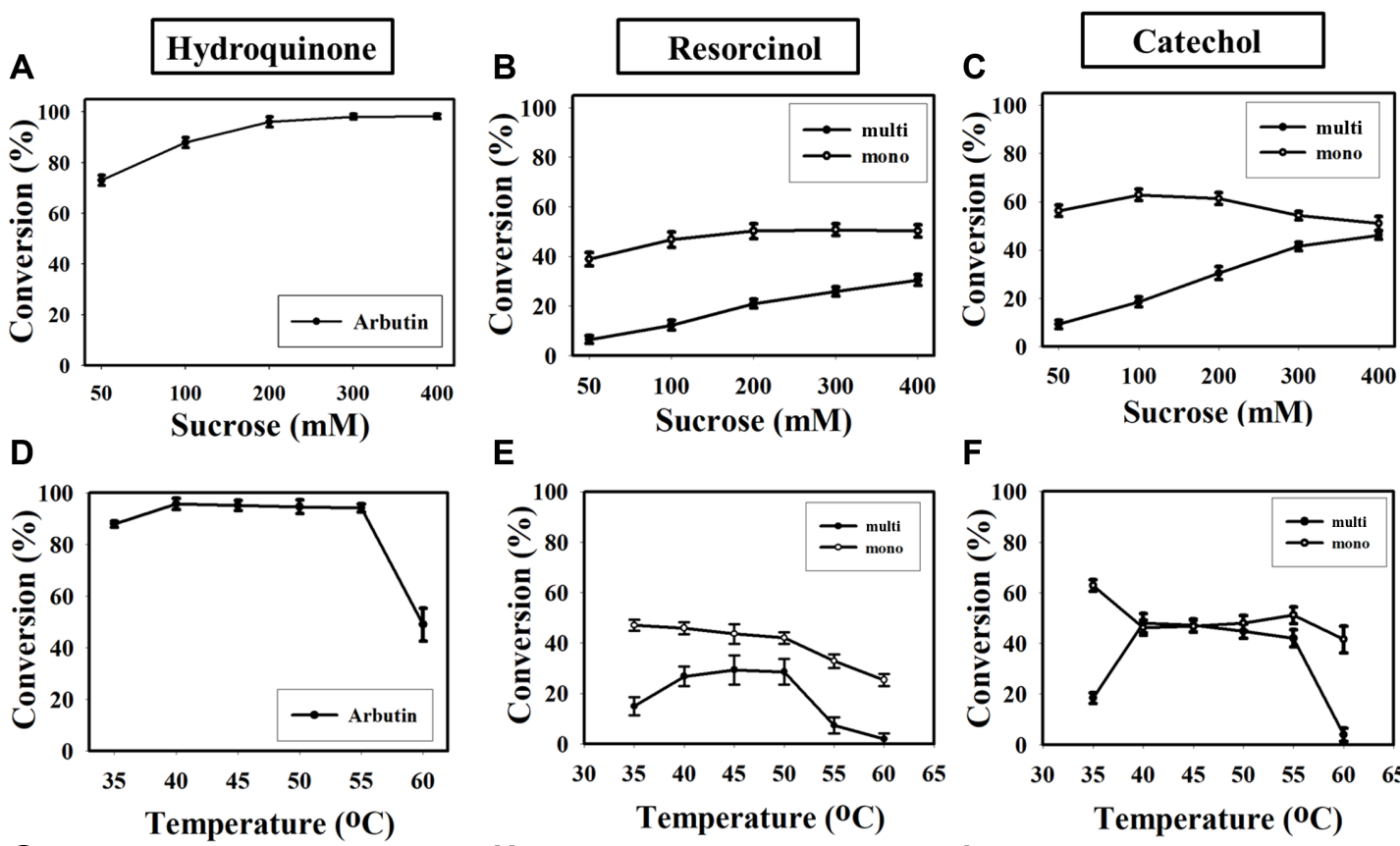

$\mathbf{F}$
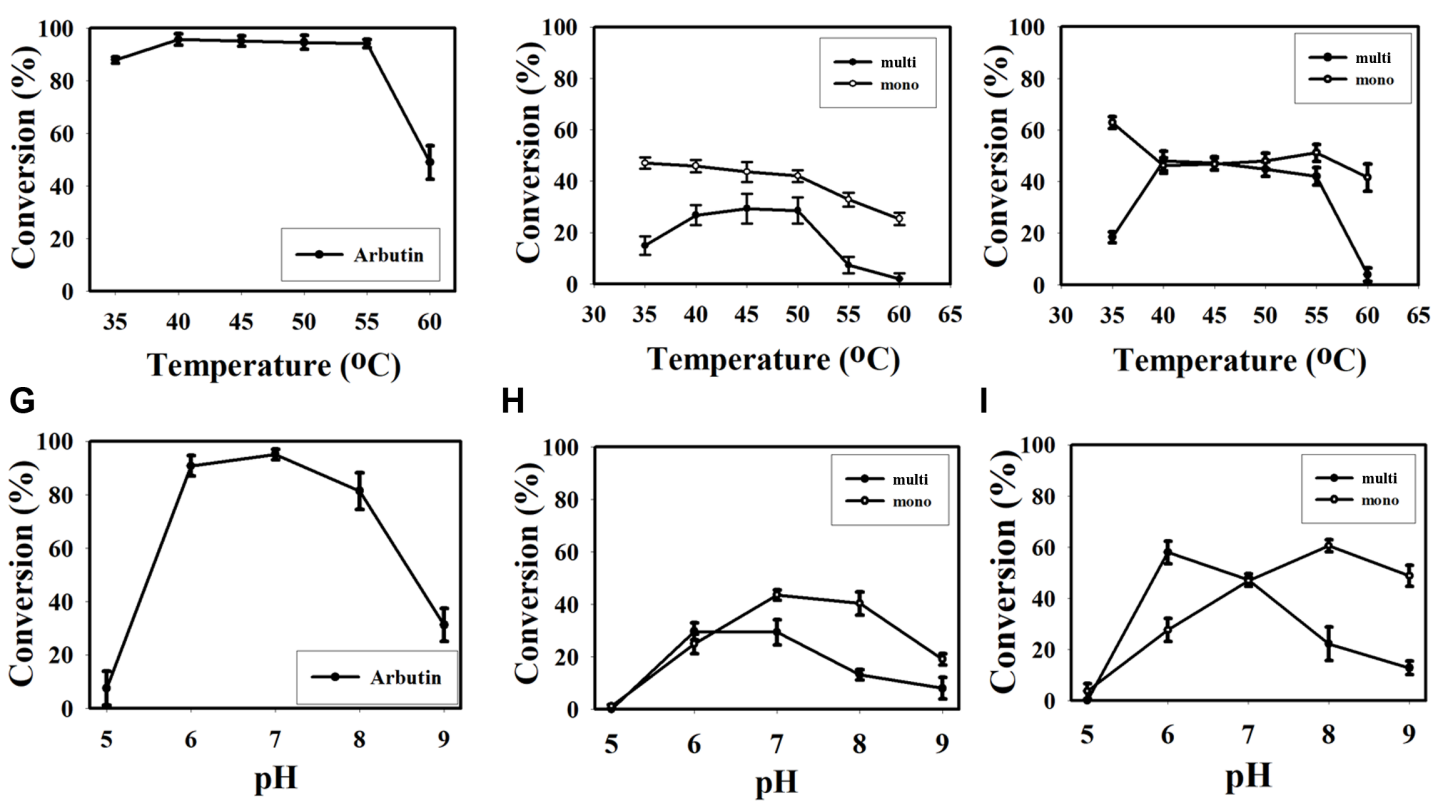

Fig. 2. Optimization of transglycosylation parameters in the reaction mixtures.

Sucrose concentration optimization in: (A) Hydroquinone reaction, (B) Resorcinol reaction and (C) Catechol reaction. Incubation temperature optimization in: (D) Hydroquinone reaction, (E) Resorcinol reaction and (F) Catechol reaction. pH optimization for (G) Hydroquinone reaction, (H) Resorcinol reaction and (I) Catechol reaction. Each reaction was performed multiple times as shown by error bars.

oligosaccharides, with extensive industrial applications for the commercial production of D-glucose from starch [18]. The same amyloglucosidase was applied for the treatment of DgAS-catalyzed reaction products to enhance the production of phenolic mono-glucosides. The enzyme from A. niger was used after the transglycosylation reaction was terminated. Approximately $13 \mathrm{U} / \mathrm{ml}$ amyloglucosidase (optimized concentration data not shown) was used to treat each reaction mixture of hydroquinone, resorcinol and catechol. The effect of glucosidase activity was directly observed by HPLC-PDA chromatographic profile during the product formation from the reaction mixtures (Figs. S3CS3F). The treatment of amyloglucosidase decreased the oligosaccharide-conjugated products while increasing the single sugar-conjugated products. However, this phenomenon was not observed in the hydroquinone reaction mixture. Most of the products were mono-glucosides (Figs. S3A and S3B). This data supported the possibility of converting multi-sugar-conjugated products to a mono-glucoseconjugated product, which results in target product enhancement.

\section{Optimization of Dihydroxybenzene Concentrations}

Finally, the production of each hydroquinone glucoside, resorcinol glucoside, and catechol glucoside, was observed under optimized conditions of incubation temperature, $\mathrm{pH}$ and sucrose concentration. In addition, the data were obtained after treatment with $13 \mathrm{U} / \mathrm{ml}$ of amyloglucosidase. 
A
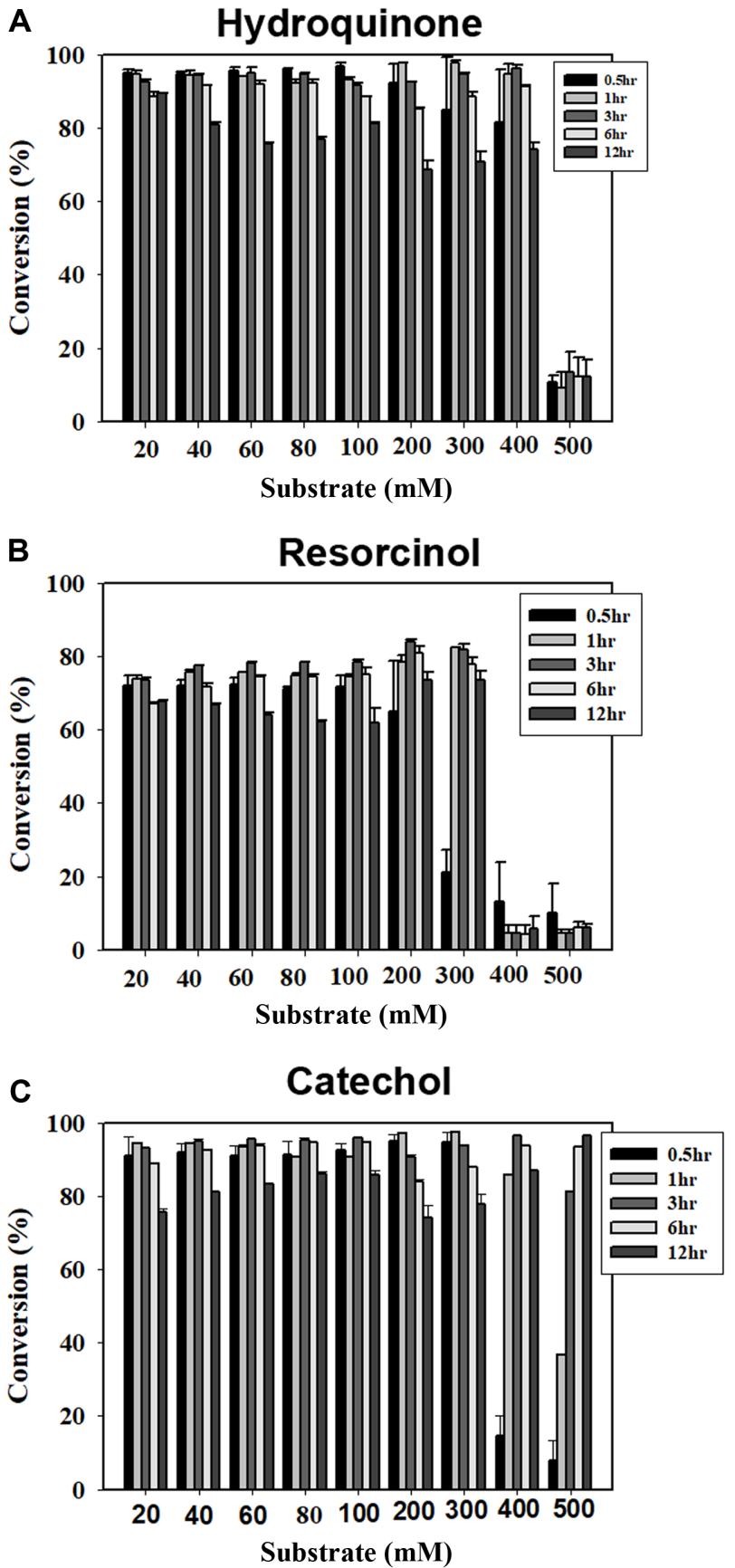

Fig. 3. Substrate concentration optimization resulted in the highest yield of hydroquinone, resorcinol and catechol glucosides.

Substrates increased in concentration from $20 \mathrm{mM}$ to $500 \mathrm{mM}$ and the reaction mixtures were analyzed by HPLC-PDA. (A) Hydroquinone concentration optimization. (B) Resorcinol concentration optimization and (C) Catechol concentration optimization.

Total reaction volume was carried out in $500 \mu \mathrm{l}$ for the transglycosylation reaction of hydroquinone. The substrate concentrations were increased from 20 to $500 \mathrm{mM}$ while maintaining other reaction components constant. We have achieved an efficient conversion of substrates into products while optimizing the sucrose concentration from 50 to $100 \mathrm{mM}$ (Figs. 2A-2C). The reactions were analyzed by HPLC-PDA at different time intervals, i.e., 0.5, 1, 3, 6, and $12 \mathrm{~h}$, respectively. The results showed that $81 \%$ of $400 \mathrm{mM}$ hydroquinone was converted to glucosides in $30 \mathrm{~min}$ of incubation time. More than $65 \%$ of $200 \mathrm{mM}$ resorcinol was converted to glucoside in $30 \mathrm{~min}$ (Figs. 3A and 3B). Similarly, at the same incubation period $94 \%$ of $300 \mathrm{mM}$ catechol was converted to products (Fig. 3C). Approximately $325.6 \mathrm{mM}$ $(88.6 \mathrm{~g} / \mathrm{l})$ of hydroquinone glucosides, $130.2 \mathrm{mM}(35.4 \mathrm{~g} / \mathrm{l})$ of resorcinol glucosides and $284.4 \mathrm{mM}(77.4 \mathrm{~g} / \mathrm{l})$ of catechol glucosides were obtained within $60 \mathrm{~min}$ of reaction. This is the highest yield of hydroquinone glucoside ( $\alpha$-arbutin), resorcinol glucoside, and catechol glucoside ever reported in such a short reaction time.

\section{Structural Confirmation}

Each reaction product was purified by prep-HPLC and subjected to NMR analyses to determine whether it was a mono-glucoside or a multiple sugar-conjugated product. The HPLC-PDA chromatogram showed distinct single peaks after purification of compounds (Fig. S4). The ${ }^{1} \mathrm{H}$ NMR signals of each glucoside product were compared with the substrates. Hydroquinone glucoside shows the anomeric signals at $5.28 \mathrm{ppm}$ with a $J$ value of $3.7 \mathrm{~Hz}$ including the presence of other sugar protons at 3.2 to $4 \mathrm{ppm}$ and confirms the product as single sugar attached at alpha configuration (Fig. 4). Resorcinol glucoside and catechol glucoside also show the anomeric signals at $5.43 \mathrm{ppm}$ with a J value of $3.7 \mathrm{~Hz}$ and at $5.28 \mathrm{ppm}$ with a J value of $3.5 \mathrm{~Hz}$ as alpha configuration.

\section{Immobilization of AS}

The enzyme immobilization experiment was performed using AMICOGEN LKZ118 beads. The immobilized enzyme was washed and reacted repeatedly 50 times for transglycosylation. In each cycle the conversion of supplied dihydroxybenzene conversion to glucosides was measured. In the case of hydroquinone, the reactivity remained constant up to 35 times on average, and the yield was maintained between $85 \%$ and $90 \%$. However, the reactivity decreased sharply after the $35^{\text {th }}$ round. On the other hand, resorcinol and catechol exhibited close to 40 -fold reactivity. The percent yield of resorcinol varied between $65 \%$ and $70 \%$ and that of catechol ranged between $85 \%$ and $90 \%$ (Fig. 5). The immobilized DgAS led to a 95, 36.9, and 


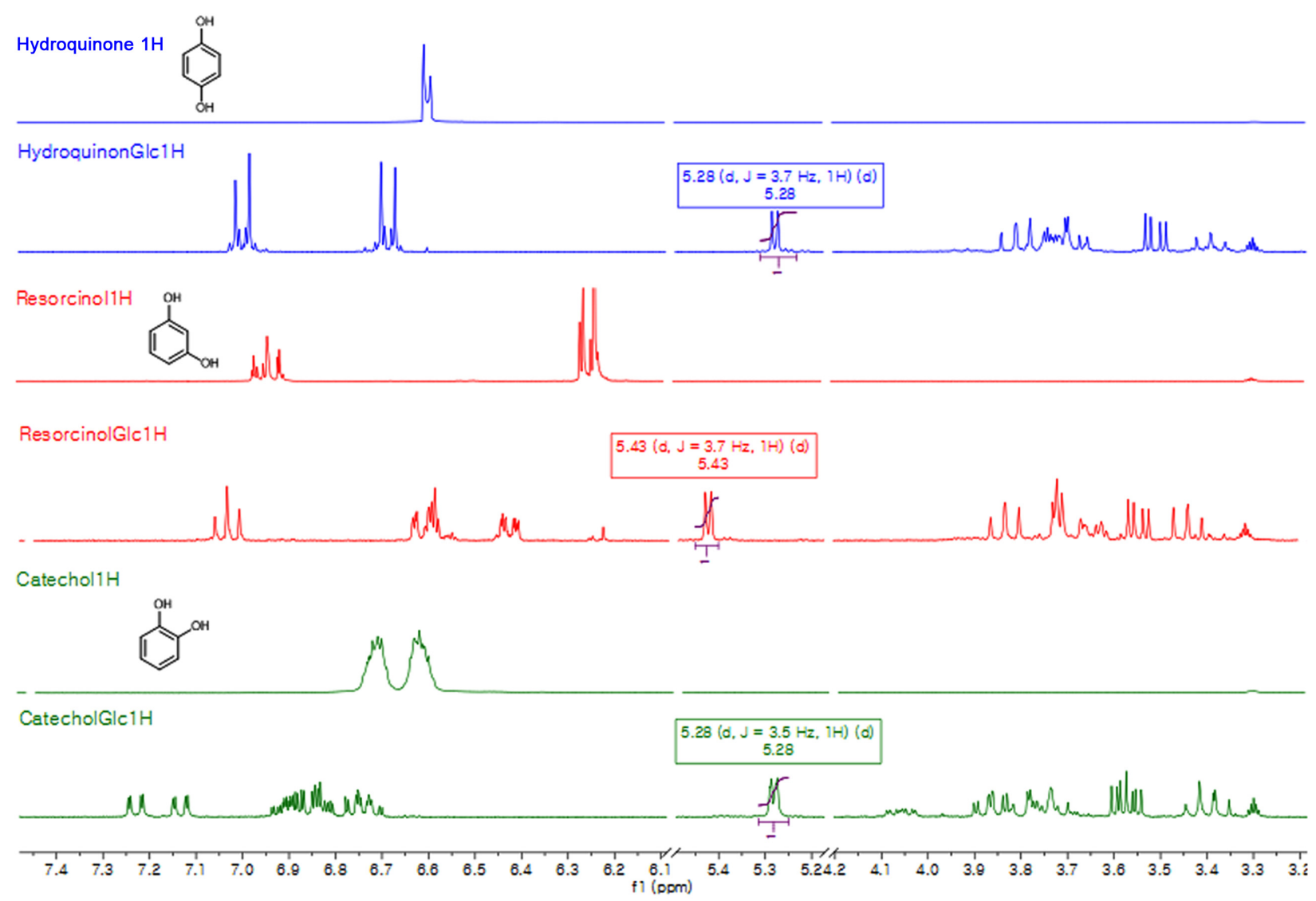

Fig. 4. ${ }^{1} \mathrm{H}$ NMR signal comparison of each substrate and glucoside

$72.2 \mathrm{~g} / 1$ final concentration of total glycosylated product of hydroquinone, catechol and resorcinol in a single cycle, respectively. Further, the efficiency of the experiments was

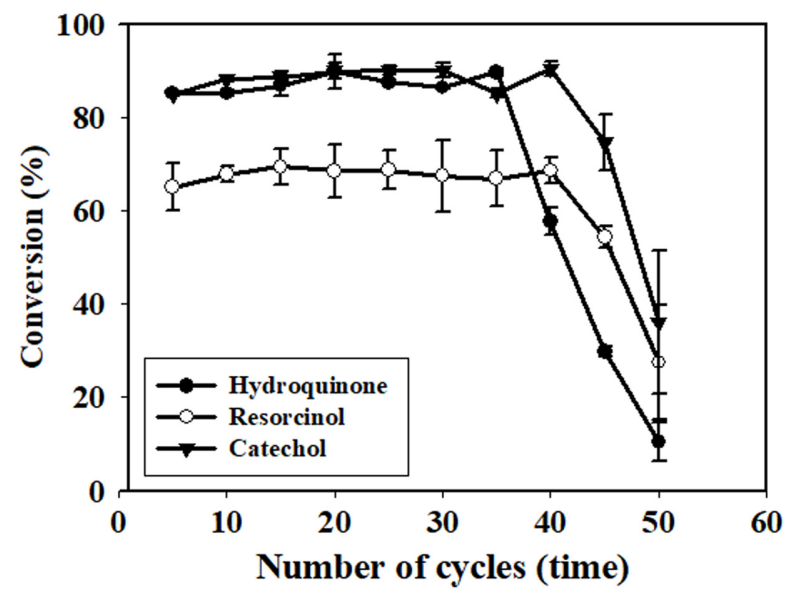

Fig. 5. Efficient use of immobilized enzymes repeatedly to generate phenolic glucosides up to 50 cycles. increased using enzyme immobilization with Amicogen LKZ118 beads, suggesting that the same immobilized enzyme can be used to synthesize phenolic glycosides inexpensively and efficiently (Fig. 5).

\section{Discussion}

Even though phenolic compounds are highly valuable, their applications in industry have been restricted due to their short life in a photosensitive environment and poor water solubility [19]. Generally, the toxicity of glycosylated product was found to be drastically lowered when compared to their aglycones [20]. Thus, transglycosylation reactions are widely employed to modify phenolic compounds such as hydroquinone, resveratrol, genistein and catechin to produce their respective glucosides [21-24]. There are several reports of transglycosylation reactions and comparative study of their biological activities with aglycones [25-28]. However, in some instances, the trace amount of substrate remained in the sample could be harmful. Thus, approaches to remove such selective toxic compounds from the reaction 
mixtures could be useful to purify the glucosylated derivatives $[29,30]$.

In this study, we generated glucosylated derivatives of three dihydroxybenzenes (hydroquinone, resorcinol and catechol) using a sustainable approach of employing immobilized amylosucrase (DgAS) and they are expected to exhibit better industrial applicability than parent compounds. In the transglycosylation reactions of biomolecules using sucrose as the sugar donor, DgAS is the most thermostable AS among various microbial sources [17]. However, all enzymes are not equally expressed in soluble fraction when they are heterologously overexpressed in E. coli systems. Thus, to express the recombinant enzyme efficiently in soluble fraction in E. coli, the codon-optimized gene is generally used to overcome the issues of protein translation and processing in soluble fractions. We have codon-optimized the nucleotide sequence of DgAS from Deinococcus geothermalis DSM 11300 and cloned it into an expression vector for protein synthesis. The transglycosylation reaction was performed using three dihydroxybenzenes. Many biocatalysts used for production of $\alpha$-arbutin have been reported and discussed recently in the presence of various sugar donors such as sucrose, maltopentose, and maltose, using the AS derived from Cellulomonas carbaniz T26 [31]. Indeed $\alpha$-arbutin has widespread applications including industrial use, as an effective skin-lightening cosmeceutical agent. However, resorcinol and catechol were not used in such catalysis and synthesis of glycoside derivatives, which explains the comparatively limited attention focused on their biological significance.

All the three derivatives of dihydroxybenzenes (hydroquinone, resorcinol and catechol) were used as substrates for transglycosylation. We targeted the synthesis of monoglucosides of each phenolic compound using sucrose as a sugar donor and AS as a biocatalyst. Based on the preliminary transglycosylation reaction, the substrate was efficiently converted to glucosides in all three reactions. Mass analysis revealed multiple sugar (gluco-oligosaccharides)-conjugated products. Hydroquinone was found to be conjugated with up to three sugars while resorcinol and catechol were linked to nearly nine sugar chains (Fig. 2). However, the target was to obtain single sugar-conjugated products of all the three phenolic compounds. Amyloglucosidase is an enzyme commercially used to obtain glucose from starch. Since this enzyme catalyzes the breakage of the $\alpha-(1,4)$ glycoside bond between the two sugars, the transglycosylation reaction mixtures were subsequently treated with commercially available amyloglucosidase to obtain monoglucosides. In this experiment, the production of sugar chain-attached glycosides was reduced while the levels of mono-glucosides increased during the HPLC-PDA chromatogram analyses (Fig. S3).

We optimized the reaction parameters to enhance productivity. Firstly, the sucrose concentration was optimized where the glucoside production increased until $400 \mathrm{mM}$ of sucrose concentration in each reaction. As shown in Fig. 2, the optimal sucrose concentration of hydroquinone, resorcinol, catechol was 300, 200, and $100 \mathrm{mM}$ (monoglucoside), respectively.

Temperature and $\mathrm{pH}$ were also optimized to $40^{\circ} \mathrm{C}$ and at $\mathrm{pH} 7$, respectively, for reaction products resulting in a higher yield. The concentration of dihydroxybenzenes was increased under the optimum conditions of sucrose concentration, temperature, and $\mathrm{pH}$. More than 95\% conversion was obtained from the hydroquinone reaction mixture while $>70 \%$ of resorcinol was converted to its glycosides. However, the catechol glycosides biosynthesis was elevated up to $100 \mathrm{mM}$ sucrose concentration with above $90 \%$ yield. This conversion percentage is substantially high for production of glycosylated product. After optimization of each reaction parameter in the transglycosylation reaction, the substrate concentration was increased and the products were analyzed at different incubation times. In each reaction mixture, the substrates were increased from 20 to $500 \mathrm{mM}$ while that of sucrose increased 5-fold resulting in the highest production of hydroquinone glucoside ( $\alpha$-arbutin), resorcinol glucoside and catechol glucoside ever reported in a such short incubation time. The products were finally purified and analyzed using NMR to confirm their identity as mono-glucosides of phenolic compounds.

The stability of enzymes often hindered their practical application in biotechnological processes. An approach to stabilize an enzyme's activity is to prevent enzyme aggregation and reduce irreversible unfolding by immobilization onto a suitable carrier [32, 33]. Immobilization of enzymes is usually performed to use them as an industrial biocatalyst. Epoxy supports are proven to be very easy to use in immobilizing enzymes [34-38]. In this article, we report a high-performing epoxy support (Amicogen LKZ118) for the immobilization of DgAS.

The transglycosylation reaction using immobilized enzyme revealed that the reactivity remained constant up to 35 cycles with hydroquinone. But, the conversion of resorcinol and catechol remained constant up to 40 cycles. Hydroquinone's earlier (5 cycles earlier than resorcinol and catechol) conversion in immobilized reaction system could be attributed to DgAS's different catalysis towards 
hydroquinone. Moreover, the hydroquinone reaction was performed with a higher dose of donor $(2 \mathrm{M})$ and acceptor $(400 \mathrm{mM})$ concentrations than used with resorcinol and catechol. This could be another reason for the early decrease in conversion percentage of hydroquinone. The immobilized DgAS led to a $278.4 \mathrm{mM}(75.8 \mathrm{~g} / \mathrm{l}), 108.8 \mathrm{mM}$ $(29.6 \mathrm{~g} / \mathrm{l})$, and $211.2 \mathrm{mM}(57.5 \mathrm{~g} / \mathrm{l})$ final concentration of mono-glycosylated hydroquinone, catechol and resorcinol at 35 cycles, respectively. This result revealed that DgAS is a stable enzyme and can be employed for conversion of large quantities of dihydroxybenzenes to glucosides by immobilizing the enzyme without decrease in any significant activity for glucoside production. This approach provides a cost-effective and easy to process immobilization (use of epoxy activated) of DgAS to generate immobilized codon optimized enzyme biocatalysts, which have great potential for industrial production of glucosylated derivatives of dihydroxybenzenes.

\section{Acknowledgments}

This work was supported by the National Research Foundation of Korea (NRF) and a grant funded by the Korea government (MEST) (NRF-2017R1A2A2A05000939).

\section{Conflict of Interest}

The authors have no financial conflicts of interest to declare.

\section{References}

1. Kim MD, Jung DH, Seo DH, Jung JH, Seo EJ, Baek NI, et al. 2016. Acceptor specificity of amylosucrase from einococcus radiopugnans and its application for synthesis of Rutin Derivatives. J. Microbiol. Biotechnol. 26: 1845-1854.

2. Overwin H, Wray V, Seeger M, Sepulveda-Boza S, Hofer B. 2016. Flavanone and isoflavone glucosylation by non-Leloir glycosyltransferases. J. Biotechnol. 233: 121-128.

3. Liang C, Zhang Y, Jia Y, Wenzhao W, Li Y, Lu S, et al. 2016. Engineering a carbohydrate-processing transglycosidase into glycosyltransferase for natural product glycodiversification. Sci. Rep. 6: 21051

4. Lim M-C, Lee G-H, Ngoc Huynh DT, Morales Letona CA, Seo D-H, Park C-S, et al. 2015. Amylosucrase-mediated synthesis and self-assembly of amylose magnetic microparticles. RSC Adv. 5: 36088-36091.

5. Letona CAM, Park CS, Kim YR. 2017. Amylosucrase-mediated beta-carotene encapsulation in amylose microparticles. Biotechnol. Prog. 33: 1640-1646.
6. O'Neill EC, Rashid AM, Stevenson CEM, Hetru A-C, Gunning AP, Rejzek M, et al. 2014. Sugar-coated sensor chip and nanoparticle surfaces for the in vitro enzymatic synthesis of starch-like materials. Chem. Sci. 5: 341-350.

7. Smit N, Vicanova J, Pavel S. 2009. The hunt for natural skinwhitening agents. Int. J. Mol. Sci. 10: 5326-5349.

8. Boer J, Jemec GB. 2010. Resorcinol peels as a possible selftreatment of painful nodules in hidradenitis suppurativa. Clin. Exp. Dermatol. 35: 36-40.

9. Limsuwan T, Boonme P, Khongkow P, Amnuaikit T. 2017. Ethosomes of phenylethyl resorcinol as vesicular delivery system for skin- lightening applications. BioMed. Res. Int. 83: 8310979.

10. Kim BS, Na YG, Choi JH, Kim I, Lee E, Kim SY, et al. 2017. The improvement of skin- whitening of phenylethylrResorcinol by nanostructured lipid carriers. Nanomaterials 7: 241.

11. Jung CT, Wickett RR, Desai PB, Bronaugh RL. 2003. In vitro and in vivo percutaneous absorption of catechol. Food Chem. Toxicol. 41: 885-895.

12. Zhang $P$, Zhang E, Xiao M, Chen C, Xu W. 2013. Enhanced chemical and biological activities of a newly biosynthesized eugenol glycoconjugate, eugenol alpha-D-glucopyranoside. Appl. Microbiol. Biotechnol. 97: 1043-1050.

13. Seo DH, Jung JH, Ha SJ, Cho HK, Jung DH, Kim TJ, et al. 2012. High-yield enzymatic bioconversion of hydroquinone to alpha-arbutin, a powerful skin- lightening agent, by amylosucrase. Appl. Microbiol. Biotechnol. 94: 1189-1197.

14. Lu L, Xu L, Guo Y, Zhang D, Qi T, Jin L, et al. 2015. Glycosylation of phenolic compounds by the site-mutated beta-galactosidase from Lactobacillus bulgaricus L3. PLoS One 10: $\mathrm{e} 0121445$.

15. Parajuli P, Pandey RP, Koirala N, Yoon YJ, Kim BG, Sohng JK. 2014. Enzymatic synthesis of epothilone A glycosides. $A M B$ Express 4: 31.

16. Pandey RP, Gurung RB, Parajuli P, Koirala N, Tuoi le T, Sohng JK. 2014. Assessing acceptor substrate promiscuity of YjiC-mediated glycosylation toward flavonoids. Carbohyd. Res. 393: 26-31.

17. Guerin F, Barbe S, Pizzut-Serin S, Potocki-Veronese G, Guieysse D, Guillet V, et al. 2012. Structural investigation of the thermostability and product specificity of amylosucrase from the bacterium Deinococcus geothermalis. J. Biol. Chem. 287: 6642-6654.

18. Selmi B, Marion D, Perrier Cornet JM, Douzals JP, Gervais P. 2000. Amyloglucosidase hydrolysis of high-pressure and thermally- gelatinized corn and wheat starches. J. Agric. Food Chem. 48: 2629-2633.

19. Garcia-Salas P, Morales-Soto A, Segura-Carretero A, Fernandez-Gutierrez A. 2010. Phenolic-compound-extraction systems for fruit and vegetable samples. Molecules 15: 88138826.

20. Suzuki Y, Uchida K. 1999. Enzymatic Glycosylation of 
Aglycones of Pharmacological Significance, pp. 297-312. In Bucke C (ed.), Carbohydrate Biotechnology Protocols, Ed. Humana Press, Totowa, NJ

21. Cho HK, Kim HH, Seo DH, Jung JH, Park JH, Baek NI, et al. 2011. Biosynthesis of (+)-catechin glycosides using recombinant amylosucrase from Deinococcus geothermalis DSM 11300. Enzyme Microb. Technol. 49: 246-253.

22. Li D, Roh SA, Shim JH, Mikami B, Baik MY, Park CS, et al. 2005. Glycosylation of genistin into soluble inclusion complex form of cyclic glucans by enzymatic modification. J. Agric. Food Chem. 53: 6516-6524.

23. Park H, Kim J, Choi KH, Hwang S, Yang SJ, Baek NI, et al. 2012. Enzymatic synthesis of piceid glucosides using maltosyltransferase from Caldicellulosiruptor bescii DSM 6725. J. Agric. Food Chem. 60: 8183-8189.

24. Seo DH, Jung JH, Ha SJ, Cho HK, Jung DH, Kim TJ, et al. 2012. High-yield enzymatic bioconversion of hydroquinone to alpha-arbutin, a powerful skin- lightening agent, by amylosucrase. Appl. Microbiol. Biotechnol. 94: 1189-1197.

25. Bae HK, Lee SB, Park CS, Shim JH, Lee HY, Kim MJ, et al. 2002. Modification of ascorbic acid using transglycosylation activity of Bacillus stearothermophilus maltogenic amylase to enhance its oxidative stability. J. Agric. Food Chem. 50: 3309-3316.

26. Rowan AS, Hamilton CJ. 2006. Recent developments in preparative enzymatic syntheses of carbohydrates. Nat. Prod. Rep. 23: 412-443.

27. Thibodeaux CJ, Melancon CE, Liu HW. 2007. Unusual sugar biosynthesis and natural product glycodiversification. Nature 446: 1008-1016.

28. Wang LX. 2008. Chemoenzymatic synthesis of glycopeptides and glycoproteins through endoglycosidase-catalyzed transglycosylation. Carbohydr. Res. 343: 1509-1522.

29. Liu C, Deng L, Zhang P, Zhang S, Xu T, Wang F, et al. 2013.
Toward a cost-effective method for $\alpha$-arbutin production by using immobilized hydroquinone as a glucosyl acceptor. Process Biochem. 48: 1447-1452.

30. Yao RS, Sun M, Wang CL, Deng SS. 2006. Degradation of phenolic compounds with hydrogen peroxide catalyzed by enzyme from Serratia marcescens AB 90027. Water Res. 40: 3091-3098.

31. Yu S, Wang Y, Tian Y, Xu W, Bai Y, Zhang T, et al. 2018. Highly efficient biosynthesis of $\alpha$-arbutin from hydroquinone by an amylosucrase from Cellulomonas carboniz. Process Biochem. 68: 93-99

32. Bryjak J, Trochimczuk AW. 2006. Immobilization of lipase and penicillin acylase on hydrophobic acrylic carriers. Enzyme Microb. Technol. 39: 573-578.

33. Kim TS, Patel SK, Selvaraj C, Jung WS, Pan CH, Kang YC, et al. 2016. A highly efficient sorbitol dehydrogenase from Gluconobacter oxydans G624 and improvement of its stability through immobilization. Sci. Rep. 6: 33438.

34. Hernaiz MJ, Crout DH. 2000. Immobilization/stabilization on Eupergit $\mathrm{C}$ of the beta-galactosidase from B. circulans and an alpha-galactosidase from Aspergillus oryzae. Enzyme Microb. Technol. 27: 26-32.

35. Katchalski-Katzir E, Kraemer DM. 2000. Eupergit ${ }^{\circledR}$ C, a carrier for immobilization of enzymes of industrial potential. J. Mol. Catal. B Enzym. 10: 157-176.

36. Boller T, Meier C, Menzler S. 2002. EUPERGIT oxirane acrylic beads: how to make enzymes fit for biocatalysis. Organ. Process Res. Dev. 6: 509-519.

37. Melander WR, Corradini D, Horvath C. 1984. Salt-mediated retention of proteins in hydrophobic-interaction chromatography: application of solvophobic theory. J. Chromatography 317: 67-85.

38. Wheatley JB, Schmidt DE, Jr. 1999. Salt-induced immobilization of affinity ligands onto epoxide-activated supports. J. Chromatography A 849: 1-12. 